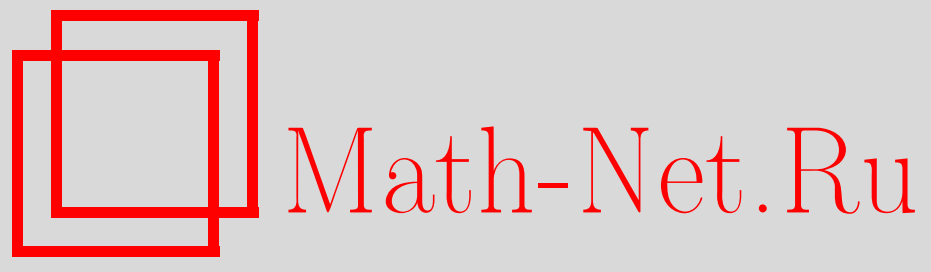

А. В. Гришин, Л. М. Цыбуля, Две теоремы о строении относительно свободной алгебры Грассмана, УМН, 2008, том 63, выпуск 4, 181-182

DOI: https://doi.org/10.4213/rm9179

Использование Общероссийского математического портала Math-Net.Ru подразумевает, что вы прочитали и согласны с пользовательским соглашением http://www . mathnet.ru/rus/agreement

Параметры загрузки:

IP : 3.85 .73 .92

26 апреля 2023 г., 12:17:01

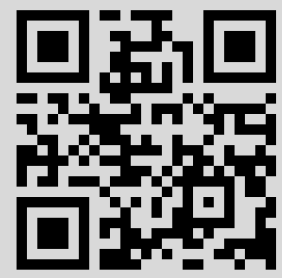




\section{Две теоремы о строении относительно свободной алгебры Грассмана}

\section{А. В. Гришин, Л. М. Цыбуля}

Пусть $F^{(3)}=k\left\langle 1, x_{1}, \ldots, x_{i}, \ldots\right\rangle / T^{(3)}$ - относительно свободная ассоциативная алгебра с единицей над бесконечным полем $k$ характеристики $p>2$, соответствующая унитарно замкнутому $T$-идеалу $T^{(3)}$, порожденному тождеством Грассмана $\left[\left[x_{1}, x_{2}\right], x_{3}\right]=0$. Образы переменных в $F^{(3)}$ обозначаются теми же буквами. На алгебре $F^{(3)}$ действует (справа) полугрупповая алгебра $k T$, где $T$ - полугруппа всех эндоморфизмов алгебры $k\left\langle 1, x_{1}, \ldots, x_{i}, \ldots\right\rangle$. По определению (см. [1]) T-пространством в $F^{(3)}$ называется любой $k T$-подмодуль. Вообще, $T$-пространство - это унитарный правый $k T$-модуль, и можно рассматривать факторпространства, прямые суммы, тензорные произведения $T$-пространств (тензорные произведения над полем $k$ с покомпонентным действием алгебры $k T$ ) и т. д.

В алгебре $F^{(3)}$ весьма важную роль играет $T$-пространство $W_{p l}$, порожденное всеми одночленами, содержащими каждую переменную с кратностью $p^{l}$ (так называемыми $p^{l}$-словами). Именно к таким $T$-пространствам сводятся многие структурные вопросы в алгебре $F^{(3)}$. Известно (см. [2]), что $W_{p^{l}}=D_{p^{l}} \oplus C D_{p^{l}}$ - прямая сумма $T$-пространств, где $D_{p^{l}}-T$-пространство, порожденное одночленом $x_{1}^{p^{l}}$, а $C D_{p^{l}}-$ $T$-пространство, порожденное всеми многочленами вида $g_{m, l}=c_{m, l} z^{p^{l}}$, где $c_{m, l}=$ $x_{1}^{p^{l}-1} y_{1}^{p^{l}-1}\left[x_{1}, y_{1}\right] \cdots x_{m}^{p^{l}-1} y_{m}^{p^{l}-1}\left[x_{m}, y_{m}\right], m, l \in \mathbb{N}, y_{i}, z$ - переменные из множества $\left\{x_{1}, \ldots, x_{i}, \ldots\right\}$, отличные от переменных $x_{1}, x_{2}, \ldots, x_{m}$. Пусть $C_{p^{l}}-T$-пространство, порожденное всеми многочленами $c_{m, l}$. Отметим, что все рассматриваемые бесконечные системы порождающих неприводимы, кроме того, $D_{p^{l}} / D_{p^{l+1}}-$ простой $k T$ модуль (см. [2]-[4]).

Введенные таким образом $T$-пространства $C_{p^{l}}$ и $C D_{p^{l}}$ раскладываются в сумму $T$-пространств $C_{p^{l}}^{(m)}$ и $C D_{p^{l}}^{(m)}$, которые порождены многочленами $c_{m, l}$ и $g_{m, l}$ соответственно (циклические $k T$-модули). Связь между введенными $T$-пространствами выражается следующей диаграммой (см. также [2], [3]), в которой, как будет видно из теоремы 1, все включения строгие:

\begin{tabular}{|c|c|c|c|c|c|c|c|c|c|c|c|}
\hline $\begin{array}{c}C D_{p} \\
\cup\end{array}$ & $=$ & $\begin{array}{c}C D_{p}^{(1)} \\
\cup\end{array}$ & + & $\begin{array}{c}C D_{p}^{(2)} \\
\cup\end{array}$ & + & $\ldots$ & + & $\begin{array}{c}C D_{p}^{(m-1)} \\
\cup\end{array}$ & + & $\begin{array}{c}C D_{p}^{(m)} \\
\cup\end{array}$ & + \\
\hline$C D_{p^{2}}$ & $=$ & $C D_{p^{2}}^{(1)}$ & + & $C D_{p^{2}}^{(2)}$ & + & $\cdots$ & + & $C D_{p^{2}}^{(m-1)}$ & + & $C D_{p^{2}}^{(m)}$ & + \\
\hline$\cup$ & & $\cup$ & & $U^{\prime}$ & & & & $\cup$ & & $\cup$ & \\
\hline$\vdots$ & & $\vdots$ & & $\vdots$ & & & & $\vdots$ & & $\vdots$ & \\
\hline$\cup$ & & $\cup$ & & $\cup$ & & & & $\cup$ & & $u$ & \\
\hline$C D_{p^{l}}$ & $=$ & $C D_{p^{l}}^{(1)}$ & + & $C D_{p^{l}}^{(2)}$ & + & $\cdots$ & + & $C D_{p^{l}}^{(m-1)}$ & + & $C D_{p^{l}}^{(m)}$ & + \\
\hline$\cup$ & & $\cup^{p}$ & & $U^{F}$ & & & & $\cup$ & & U & \\
\hline$\vdots$ & & $\vdots$ & & $\vdots$ & & & & $\vdots$ & & $\vdots$ & \\
\hline$\cup$ & & $U$ & & $\cup$ & & & & $\cup$ & & $u$ & \\
\hline $\begin{array}{c}C_{p^{l}} \\
\cup\end{array}$ & $=$ & $\begin{array}{c}C_{p^{l}}^{(1)} \\
\cup\end{array}$ & + & $\begin{array}{c}C_{p^{l}}^{(2)} \\
\cup\end{array}$ & + & $\ldots$ & + & $\begin{array}{c}C_{\left.p^{l}-1\right)}^{(m-1)} \\
\cup\end{array}$ & + & $C_{p^{l}}^{(m)}$ & + \\
\hline$\vdots$ & & $\vdots$ & & : & & & & $\vdots$ & & $\vdots$ & \\
\hline$\cup$ & & $u$ & & $\cup$ & & & & $U$ & & $\cup$ & \\
\hline $\begin{array}{l}C_{p} \\
\cup\end{array}$ & $=$ & $C_{p}^{(1)}$ & + & $C_{p}^{(2)}$ & + & $\cdots$ & + & $\begin{array}{c}C_{p}^{(m-1)} \\
\cup\end{array}$ & + & $C_{p}^{(m)}$ & + \\
\hline$C_{1}$ & $=$ & $C_{1}^{(1)}$ & $\supset$ & $C_{1}^{(2)}$ & $\supset$ & $\ldots$ & $\supset$ & $C_{1}^{(m-1)}$ & $\supset$ & $C_{1}^{(m)}$ & $\supset$ \\
\hline
\end{tabular}

Работа выполнена при поддержке РФФИ (грант № 07-01-00625). 
Здесь $C_{1}^{(m)}-T$-пространство, порожденное произведением $m$ коммутаторов.

Пусть $\overline{C D_{p^{l}}}=C D_{p^{l}} / C D_{p^{l+1}}$. Тогда $\overline{C D_{p^{l}}}=\sum_{r=1}^{\infty} \overline{C D_{p^{l}}^{(r)}}$, где $\overline{C D_{p^{l}}^{(r)}}-$ образ $C D_{p^{l}}^{(r)}$ в $T$-пространстве $\overline{C D_{p^{l}}}$. Положим $\Delta_{p^{l}}^{(m)}=\sum_{r=m}^{\infty} \overline{C D_{p^{l}}^{(r)}}$. Имеется бесконечная строго убывающая цепочка $\overline{C D_{p^{l}}}=\Delta_{p^{l}}^{(1)} \supset \Delta_{p^{l}}^{(2)} \supset \cdots$, причем $\bigcap_{m} \Delta_{p^{l}}^{(m)}=0$ для любого $l \in \mathbb{N}$. Положим $\Omega\left(C D_{p^{l}}^{(m)}\right)=C D_{p^{l+1}}^{(m)}+\sum_{i \neq m} C D_{p}^{(i)}, \Omega\left(C_{p^{l}}^{(m)}\right)=C_{p^{l-1}}^{(m)}+$ $\sum_{i \neq m} C D_{p}^{(i)}$, где $l, m \in \mathbb{N}$. Другими словами, если $M$ равно $C_{p^{l}}^{(m)}$ или $C D_{p^{l}}^{(m)}$, то $\Omega(M)$ - $T$-пространство, порожденное всеми $T$-пространствами, находящимися в приведенной диаграмме ниже $M$ (достаточно взять ближайшее снизу), а также находящимися левее и правее $M$ (достаточно взять самые верхние).

Некоторую независимость любого элемента $C_{p^{l}}^{(m)}$ или $C D_{p^{l}}^{(m)}$ из приведенной выше диаграммы от всех остальных, кроме тех, что находятся выше по столбцу, показывает

Теорема 1. а) Пусть $M$ равно $C_{p^{l}}^{(m)}$ или $C D_{p^{l}}^{(m)}$. Тогда $(M+\Omega(M)) / \Omega(M)$ - однопорожденное ненулевое T-пространство и, следовательно, однопорожденные T-пространства $C_{p^{l}}^{(m)} / C_{p^{l-1}}^{(m)}$ и $C D_{p^{l}}^{(m)} / C D_{p^{l+1}}^{(m)}$ ненулевые.

б) Фактор-Т-пространство $\Delta_{p^{l}}^{(m)} / \Delta_{p^{l}}^{(m+1)}$ является ненулевым гомоморфным образом тензорного произведения набора $2 m+1$ копий простого T-пространства $D_{p^{l}} / D_{p^{l+1}}$ (имеется и более точное описание, которое здесь не приводится).

Можно показать (см. [3], [5]), что $T$-пространство $W_{p}$ является подалгеброй $k$-алгебры $F^{(3)}$, лежащей в ее центре (вопрос о совпадении с центром пока открыт), причем $C D_{p}-$ радикал алгебры $W_{p}$, являющийся ненильпотентной ниль-алгеброй индекса $p$, и $W_{p} / C D_{p} \cong D_{p}$ - алгебра коммутативных многочленов $k\left[x_{1}^{p}, \ldots, x_{i}^{p}, \ldots\right]$. Строение $W_{p}$-модулей $F^{(3)}$ и $C D_{p}$ в некоторой степени проясняет следующая

TEOPEмA 2. а) Пусть $u\left(m_{i_{1}}, \ldots, m_{i_{r}}\right)=x_{i_{1}}^{m_{i_{1}}} \ldots x_{i_{r}}^{m_{i_{r}}}$, где $i_{1}<\cdots<i_{r}, 1 \leqslant m_{i_{\alpha}} \leqslant$ $p-1, S$ - множество, состоящее из 1 и всех одночленов $u\left(m_{i_{1}}, \ldots, m_{i_{r}}\right)$. Тогда

$$
F^{(3)}=W_{p} \cdot S=W_{p} \cdot 1+\sum_{\left(m_{i_{1}}, \ldots, m_{i_{r}}\right)} W_{p} u\left(m_{i_{1}}, \ldots, m_{i_{r}}\right),
$$

причем $S$ - бесконечная неприводимая система порождающих $W_{p}$-модуля $F^{(3)}$.

б) Пусть $L$ - множество многочленов, получающихся из многочлена $c_{1,1}$ c помощъю линейных подстановок, $k$-линейных действий и подстановок вместо переменных элементов из системы $S$. Тогда $C D_{p}=W_{p} \cdot L$ - бесконечно порожденный $W_{p}$-модуль.

в) $F^{(3)} u W_{p}$ - свободные $D_{p}$-модули бесконечного ранга.

ЗАмечАния. (i) Соотношения между элементами системы порождающих $S$ можно полностью описать. (iі) В случае $p=2$ имеются аналоги приведенных результатов; однако есть и существенные особенности.

\section{Список литературы}

[1] А. В. Гришин, Фундамент. и прикл. матем., 5:1 (1999), 101-118. [2] А. В. Гришин, Л. М. Сурмина, УМН, 62:4 (2007), 145-146. [3] А. В. Гришин, Л. М. Цыбуля, Вестник СамГУ. Сер. матем., 57:7 (2007), 35-55. [4] В. В. Щиголев, Матем. сб., 191:3 (2000), 143-160. [5] А. В. Гришин, Л. М. Сурмина, Тезисы докладов Междунар. конф. по алгебре и теории чисел, посв. 80-летию В.Е. Воскресенского (Самара, 2007), 15-16.

А. В. Гришин (А. V. Grishin)

Московский педагогический государственный университет

Л. М. Цыбуля (L. M. Tsybulya)

Московский педагогический государственный университет

E-mail: liliya-kinder@mail.ru
Представлено А. В. Михалёвым Принято редколлегией 25.01.2008 